\title{
COMPARAÇÃO DE PRODUTOS DE RADIAÇÃO SOLAR INCIDENTE À SUPERFÍCIE PARAA AMÉRICA DO SUL
}

\section{LUCÍA IRACEMA CHIPPONELLI PINTO ${ }^{1}$, MARCOS HEIL COSTA ${ }^{2}$, LUCIANA MARA FREITAS DINIZ ${ }^{3}$, GILBERTO C. SEDIYAMA ${ }^{2}$ E FERNANDO FALCO PRUSKI ${ }^{2}$}

\author{
${ }^{1}$ Centro de Ciências do Sistema Terrestre/Instituto Nacional de Pesquisas Espaciais (CCST/INPE), São Jose \\ dos Campos, SP, Brasil \\ ${ }^{2}$ Universidade Federal de Viçosa (UFV), Departamento de Engenharia Agrícola, Viçosa, MG, Brasil \\ ${ }^{3}$ Universidade Federal de Minas Gerais (UFMG), Departamento de Ciência da Computação, Belo Horizonte, \\ MG, Brasil \\ lucia.chipponelli@dpi.inpe.br
}

Recebido Agosto 2008 - Aceito Abril 2010

\begin{abstract}
RESUMO
Para algumas aplicações meteorológicas, o conhecimento da radiação solar incidente à superfície $\left(\mathrm{S}_{\text {in }}\right)$ é muito importante. Métodos convencionais de medida deste fluxo são pontuais e, portanto, representativos de pequenas áreas. Por outro lado, os satélites meteorológicos possibilitam uma cobertura regular de todo o globo terrestre, proporcionando estimativas de fluxos radiativos sobre grandes áreas com resolução espacial do sensor (apenas alguns quilômetros). Além disso, produtos de reanálise fornecem estimativas alternativas de $S_{\text {in }}$, que precisam ser adequadamente validadas. Este trabalho compara quatro bancos de dados de radiação solar incidente para a América do Sul: três produtos provenientes de reanálise (NCEP/NCAR, ERA-40 e Eta/CPTEC) e o produto gerado pelo algoritmo GL1.2, que produz estimativas da $S_{\text {in }}$ a partir do conjunto de imagens do satélite GOES. Os resultados mostram que os campos de radiação solar incidente dos produtos de reanálise do NCEP/NCAR, ERA-40 e as estimativas do satélite GOES apresentam valores médios mensais bem próximos para todos os biomas e bacias estudados. Já a reanálise do Eta/CPTEC apresentou valores bem mais elevados para à radiação sobre a América do Sul, tanto para a média anual como nas médias mensais analisadas.
\end{abstract}

Palavras-Chave: Radiação solar incidente, Reanálise, Série de dados

\begin{abstract}
PRODUCT COMPARISON OF SOLAR RADIATION INCIDENT SURFACE FOR SOUTH AMERICA

For some meteorological applications, knowledge of incoming solar radiation at surface level $\left(\mathrm{S}_{\text {in }}\right)$ is very important. Conventional measurements of this flux by individual sensors are representative of small areas only. On the other hand, meteorological satellites allow a regular cover of the entire globe, providing estimates of radiative fluxes over wide areas, within the spatial resolution of the sensor (a few $\mathrm{km}$ ). In addition, reanalysis products provide alternative $S_{\text {in }}$ estimates that must be adequately validated. This work compares four long-term $S_{\text {in }}$ databases for South America: three reanalysis products (NCEP/NCAR, ERA-40 e Eta/CPTEC) and the database produced by the algorithm GL1.2, which estimates $\mathrm{S}_{\text {in }}$ from a set of GOES images. The results indicate that the monthly fields of the NCEP/NCAR and the ERA-40 and the GOES-based estimates are very similar for all studied regions. On the other hand, Eta/CPTEC reanalysis presents much higher radiation values, for both the long-term annual and monthly means over South America.
\end{abstract}

Keywords: Solar Radiation Incident, Reanalysis, datasets. 


\section{INTRODUÇÃO}

Para algumas aplicações meteorológicas, particularmente as aplicações na agricultura, o conhecimento do balanço de radiação à superfície $\left(\mathrm{S}_{\text {in }}\right)$ é necessário, assim como é para a modelagem (por exemplo, modelos hidrológicos, modelos de dinâmica de vegetação, entre outros) e previsão a curto e longo prazo. Métodos convencionais de medida deste balanço são pontuais e, portanto, representativos de pequenas áreas. Por outro lado, os satélites meteorológicos possibilitam uma cobertura regular de todo o globo terrestre, o que seria impossível apenas com as estações de superfície; desta forma, esses satélites oferecem um potencial de medida de fluxos radiativos sobre grandes áreas com resolução espacial do sensor (alguns quilômetros), e são fundamentais para o monitoramento do balanço de radiação à superfície no tempo e no espaço.

Existem esforços de diversos pesquisadores para estimar as componentes radiativas e o balanço radiativo no topo da atmosfera e/ou à superfície, utilizando modelos matemáticos e dados de satélite. De um modo geral os pesquisadores têm utilizado códigos complexos de transferência radiativa (Zhang et al. 1995; Rossow e Zhang, 1995, dentre outros), assim como modelos físicos simplificados para estimar as componentes radiativas (Ceballos et al. 2004), ou realizam análises a partir de dados obtidos em experimentos de campo (Calvet e Viswanadham, 1993; Galvão, 1999; Correia, 2000).

Movidos pela necessidade de solucionar a carência de observações das variáveis meteorológicas, alguns cientistas perceberam que o advento da modelagem numérica poderia se tornar uma ferramenta eficiente para gerar séries históricas das variáveis meteorológicas, com a vantagem de possibilitar sua expansão para regiões mais remotas do globo, onde a observação convencional das variáveis meteorológicas é difícil de ser realizada. Fundamentados nesta possibilidade, centros de meteorologia e de estudos climáticos criaram produtos para suprir tais dificuldades, gerando bancos de dados através de modelos numéricos, sendo a reanálise um dos produtos obtido a partir da modelagem numérica, sendo atualmente de extrema importância para a comunidade científica. As séries históricas de reanálise, geradas a partir de modelos, são construídas com base em séries de variáveis meteorológicas disponíveis a partir de algum tipo de observação.

A técnica de reanálise atmosférica processa, simultaneamente, uma grande variedade de observações de dados meteorológicos [observados em superfície (estações), aviões, navios, imagens de satélite, imagens de radar e radiossondagens], usando as leis físicas contidas nos modelos de previsão do tempo. Sendo assim, o histórico do estado atmosférico pode ser reconstruído dinamicamente. A quantidade e a qualidade dos dados observados usados para reanálise são cruciais. Por isso, é necessário fazer um controle de qualidade dos mesmos antes da assimilação visando reduzir os erros na reanálise.

As séries de dados de reanálise apresentam algumas vantagens em relação a outros produtos de séries históricas de dados. Nelas, a maioria das variáveis é consistente, obedecendo às leis físicas básicas do modelo de previsão numérica e a qualidade é controlada, eliminando descontinuidades nas séries em função das mudanças no sistema de assimilação que contêm erros instrumentais ou do observador. Os produtos de reanálise se sobrepõem aos climatológicos, principalmente por abrangerem longos períodos e pela quantidade de variáveis, de superfície ou altitude, que são fornecidas. Isto difere dos dados climatológicos observados, que são em grande parte de superfície. Portanto, o sucesso das séries de reanálise pode ser atribuído ao número, variedade e qualidade das aplicações de seus produtos.

Pohlmann e Greatbatch (2006), entretanto, mostram que as diferentes reanálises freqüentemente apresentam discordância entre si, principalmente em regiões onde as observações são escassas, como por exemplo, o hemisfério sul, onde há pouca disponibilidade de dados até os dias atuais.

Modelos físicos elaborados para estimar a radiação solar à superfície propõem relações entre fluxos no topo e à superfície, baseadas em equações de transporte radiativo (Ceballos e Moura, 1997). Nesses modelos, os parâmetros dependem das condições atmosféricas. Portanto, são dependentes de algumas variáveis climáticas regionais e com algumas ressalvas podem ser generalizados para áreas extensas. Os dados de uma rede solarimétrica servem principalmente para conferir a qualidade do método de estimativa. O algoritmo GL teve sua primeira versão foi desenvolvida na Universidade Federal da Paraíba (UFPB) para imagens do canal visível (VIS) do satélite Meteosat 4, e adaptada para o canal VIS do satélite GOES (Geostationary Operational Environmental Satellite) por pesquisadores da Divisão de Satélites e Sistemas Ambientais/Instituto Nacional de Pesquisas Espaciais (DSA/INPE) e UFPB (Bastos et al. 1996). Uma versão atualizada deste algoritmo, GL1.2, foi instalada no Centro de Previsão de Tempo e Estudos Climáticos (CPTEC) com algumas modificações, fornecendo distribuições diárias, semanais e mensais de irradiação solar global (Ceballos et al., 2004).

O objetivo deste trabalho é comparar as médias de longo prazo da radiação solar incidente à superfície, produzidas por diferentes reanálise (NCEP/NCAR, ERA-40/ECMWF, Eta/ CPTEC) com o produto elaborado pelo algoritmo GL1.2 (Ceballos et al., 2004), usando este último como referência. Serão analisados a distribuição espacial dos campos desta variável, e a sua variação meridional. Para fins de recomendação de uso por modelos hidrológicos e modelos ecológicos, os produtos também foram comparados para diferentes tipos de vegetação (floresta tropical, cerrado, caatinga e pampas/campos) 
e para as principais bacias hidrográficas (Amazônica, Orinoco, Tocantins/Araguaia, São Francisco, Paraná/Prata e Patagônia).

\section{DADOS E METODOLOGIA}

\subsection{Dados}

Foram comparados quatro bancos de dados, sendo três bancos de dados de reanálise (NCEP/NCAR, ERA-40 e Eta/ CPTEC) e um baseado em dados de sensoriamento remoto via satélite para a América do Sul (GL1.2).

A reanálise regional Eta/CPTEC é resultado da combinação de previsões diárias de curto prazo reprocessadas, em alta resolução temporal e espacial, e de dados reais observados. Estas previsões são decorrentes do modelo regional de mesoescala Eta/CPTEC. Este modelo se propõe a prever com maior detalhe fenômenos associados a sistemas de mesoescala, como frentes, brisa marítima, tempestades severas, etc.

O modelo Eta é proveniente do National Centers for Environmental Prediction (NCEP) (Mesinger et al., 1988; Black, 1994). O modelo tem sido utilizado operacionalmente para produzir previsões de tempo para a América do Sul desde 1996 no INPE. É um modelo em ponto de grade, na horizontal, as variáveis são distribuídas na grade $\mathrm{E}$ de Arakawa (Arakawa e Lamb, 1977), e a coordenada vertical é a coordenada $\eta$ (letra grega Eta, que dá o nome ao modelo). O topo do modelo está em $25 \mathrm{hPa}$, o esquema de integração é por particionamento explícito ('split-explicit'). Os termos de ajuste devido às ondas de gravidade são tratados pelo esquema 'foward backward' modificado por Janji' C (1979). O esquema de diferenças finitas no espaço suprime a separação das ondas de gravidade das duas sub-grades C (Mesinger, 1974; Janji'C, 1979). A difusão horizontal é não-linear de $2^{\mathrm{a}}$ ordem. A condição de contorno lateral é prescrita em uma fileira, sem necessidade de relaxação da lateral.

A versão do modelo Eta/CPTEC utilizado para criar a reanálise Eta/CPTEC, é hidrostático, com resolução horizontal de $40 \mathrm{~km}$ e vertical de 38 níveis. Este modelo é processado para a América do Sul e oceanos adjacentes, a partir das condições de contorno da análise global do modelo do NCEP de 6 em 6 horas, temperatura da superfície do mar semanal e neve diária.

O projeto de reanálise do National Centers for Environmental Prediction/National Center for Atmospheric Research (NCEP/NCAR) começou em 1991, como um resultado do projeto do CDAS (Climate Data Assimilation System). A idéia básica deste projeto é usar um sistema fixo de análise/ previsão e executar assimilação de dados passados, de 1948 até o presente. O modelo global espectral é rodado usando a resolução espectral T62 (209 km) e 28 níveis verticais (Kalnay et al.,
1996). Nos últimos anos algumas melhorias foram implantadas, como, por exemplo, um melhor esquema de diagnóstico de nuvens, resultando em uma melhoria na predição de radiação de ondas longas.

O European Center for Medium-Range Weather Forecasts (ECMWF) disponibiliza dois produtos de reanálise global, denominados de ERA-15 e ERA-40, para diversas variáveis meteorológicas. O ERA-40 é uma série de dados de segunda geração, que através dos avanços ocorridos nos recursos computacionais veio complementar o ERA-15. Os dados contêm valores diários e mensais da análise e previsão para um período de aproximadamente 45 anos (1957 a 2002), sendo estes interpolados em uma grade gaussiana com 60 níveis verticais e tendo uma resolução de $2,5^{\circ}$ x $2,5^{\circ}$ (lat/long), gerando quatro campos de análise por dia (00, 06, 12 e 18 UTC) (Kållberg et al., 2004).

O algoritmo físico GL1.2 é utilizado para gerar estimativas de radiação solar através destas imagens de satélite GOES. Este algoritmo supõe a partição do espectro solar em apenas dois intervalos espectrais de banda larga: 1) ultravioleta + visível, e 2) infravermelho próximo, e não considera o efeito de aerossóis. As estimativas de nebulosidade são cruciais para todos os algoritmos que utilizam dados de satélite, já que as nuvens são as principais moduladoras da radiação solar. A avaliação correta do fator de refletância máximo e mínimo das mesmas é tratada com muito cuidado por este modelo (Ceballos et al., 2004). A resolução dos dados gerados é de $0,4^{\circ} \times 0,4^{\circ}$ (lat/ lon), e a aplicação das matrizes do GL1.2 permite caracterizar de forma bastante clara comportamentos regionais de radiação solar incidente para a América do Sul.

\subsection{Metodologia}

Os quatro bancos de dados foram trabalhados no NCL (NCAR Command Language), que é uma linguagem projetada especificamente para o processamento e visualização de dados científicos. Antes de tudo, fez-se uma análise detalhada das condições dos arquivos de dados, em termos de resolução, período, formato e região de análise. Assim, os dados que não se encontravam em formato netCDF foram convertidos para este formato, antes de serem trabalhados no NCL (Tabela 1).

Para realizar esta análise da radiação solar incidente à superfície, todos os bancos de dados foram submetidos a máscaras, em que os valores de radiação solar incidente foram plotados somente no continente e, em seguida, separados por bandas de latitude desde os $10^{\circ} \mathrm{N}$ até $50^{\circ} \mathrm{S}$, variando de $5^{\circ} \mathrm{em}$ $5^{\circ}$, resultando em um total de 12 bandas para o continente. Com as bandas prontas calculou-se a média, utilizando os "scripts" do NCL, para cada uma das cinco séries de dados de radiação avaliadas neste estudo. 
A máscara utilizada para diferenciar os tipos de vegetação que predominam na América do Sul foi criada pelo Center for Sustainability and the Global Environment da University of Wisconsin-Madison (www.sage.wisc.edu). Ao todo são representados 15 tipos de biomas para o globo todo, na resolução de $0,5^{\circ} \times 0,5^{\circ}$ (lat/long).

A partir da máscara original foram criadas outras máscaras, porém com resoluções diferentes $\left(1,0^{\circ} \times 1,0^{\circ}\right.$ e $2,5^{\circ}$ x $2,5^{\circ}$ ). Para fazer essa modificação de resolução foi adotado o critério de maior concentração de bioma na célula original $\left(0,5^{\circ} \times 0,5^{\circ}\right)$. Por exemplo, para converter de $0,5^{\circ}$ para $2,5^{\circ}$ criou-se uma matriz de 5 por 5 , e tomou-se a condição de que

Tabela 1 - Informações dos produtos utilizados.

\begin{tabular}{lccc}
\hline \multicolumn{1}{c}{ Produtos } & Resolução & $\begin{array}{c}\text { Formato } \\
\text { Original }\end{array}$ & Período \\
\hline Eta/CPTEC & $0,4^{\circ} \times 0,4^{\circ}$ & Grib & $2000-2004$ \\
NCEP/NCAR & $\sim 1,9^{\circ} \times 1,9^{\circ}$ & netCDF & $1948-2006$ \\
ERA-40 & $2,5^{\circ} \times 2,5^{\circ}$ & netCDF & $1958-2002$ \\
GL1.2(GOES) & $0,4^{\circ} \times 0,4^{\circ}$ & Bin & $1997-2004$ \\
\hline
\end{tabular}

o bioma que predominasse em mais de $50 \%$ representaria o valor da nova célula $\left(2,5^{\circ} \times 2,5^{\circ}\right)$. Procedimento semelhante foi realizado para criar a máscara de $1,0^{\circ} \times 1,0^{\circ}$. Em seguida, as máscaras (Figura 1) foram aplicadas aos dados, possibilitando calcular a média mensal de cada bioma. É importante ressaltar, que ao fazer a conversão de resolução das máscaras, perde-se um pouco da qualidade da mesma, já que pontos de grade que pertenciam a um determinado bioma na máscara original, ao serem convertidos para outra resolução podem ser classificados como outro tipo de vegetação.

Da mesma forma que foi feita a análise para os tipos de vegetação, foram realizadas análises com a máscara das bacias hidrográficas das América do Sul, obtidas do projeto R-Hydronet (http://www.r-hydronet.sr.unh.edu). Os dados possuem formato ASCII GRID, que é um formato de arquivo texto, onde eles se encontram no sistema de coordenadas geográficas (lat/lon), cada valor representando um código para a identificação da bacia, para cada pixel de $0,5^{\circ} \times 0,5^{\circ}$ de resolução. Também foi necessário criar máscaras novas, compatíveis com as resoluções de $1,0^{\circ} \times 1,0^{\circ}$ e $2,5^{\circ} \times 2,5^{\circ}$. O procedimento adotado foi o mesmo aplicado ao tratamento tipo de vegetação. A Figura 2 ilustra as principais bacias hidrográficas da América do Sul.

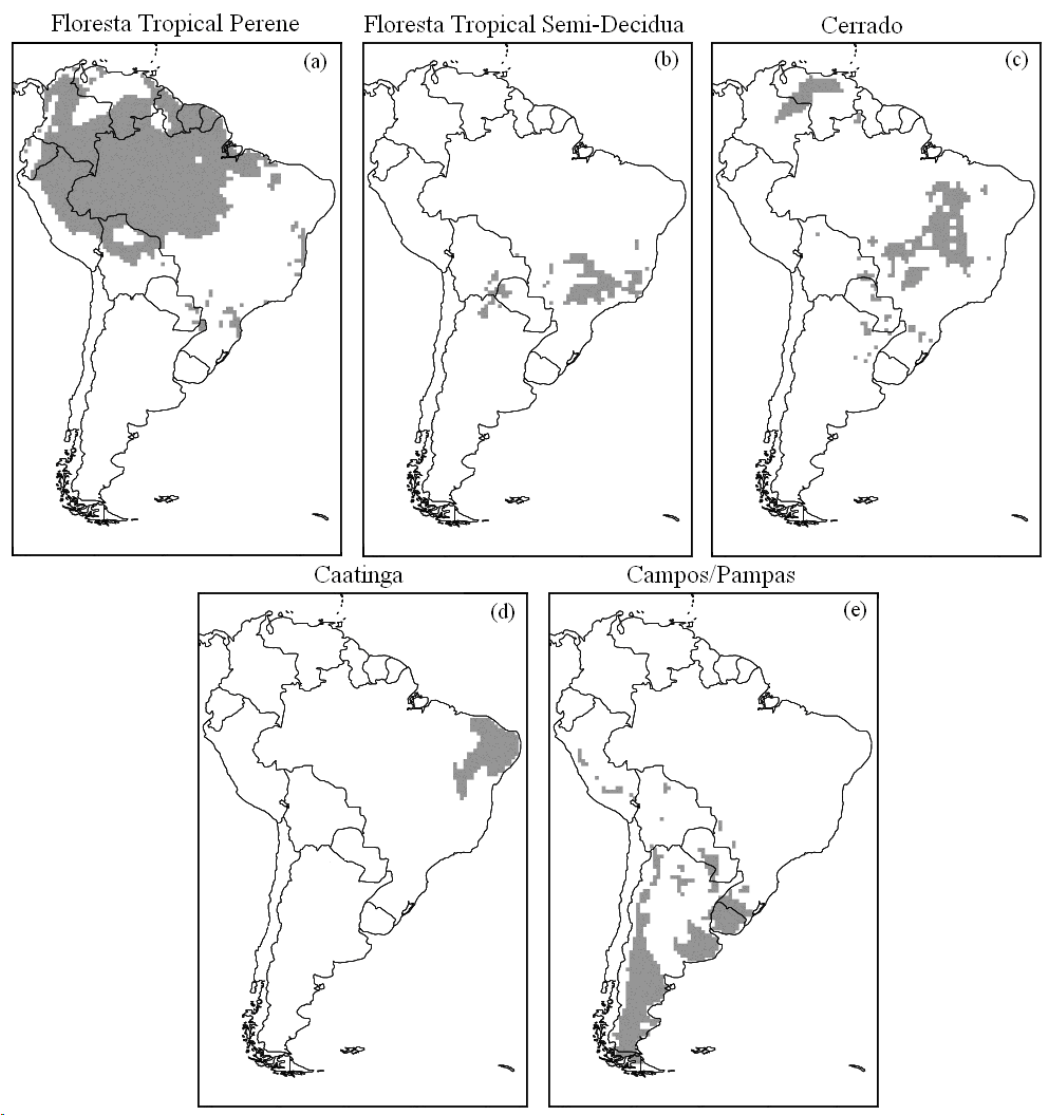

Figura 1 - Máscaras com os tipos de vegetação adotados, (a) Floresta Tropical Perene, (b) Floresta Tropical Semi-Decídua, (c) Cerrado, (d) Caatinga e (e) Campos/Pampas. 


\section{RESULTADOS E DISCUSSÃO}

\subsection{Comparação da Média Diária Anual de Radiação Solar à Superfície}

Os campos com as climatologias (médias anuais) dos produtos de reanálise (Figura 3), mostram que a América do Sul é um continente com altos valores de radiação solar incidente, em sua maior parte. Nesta figura, fica bem realçado que o continente possui uma média anual em torno de $200 \mathrm{~W} / \mathrm{m}^{2}$ (Figura 3b) [para converter de $\mathrm{W} / \mathrm{m}^{2}$ para $\mathrm{MJ} /\left(\mathrm{m}^{2}\right.$.dia), multiplica-se pelo fator de conversão de 0,0864]. O algoritmo GL1.2 (Figura 3b), baseado em dados de imagens de satélite, apresenta valores médios de radiação, para a maior parte do continente, entre 200 e $250 \mathrm{~W} / \mathrm{m}^{2}$. Os valores mais altos deste produto estão sobre a fronteira norte do continente com o oceano Atlântico, onde se verifica uma faixa com valores de $250 \mathrm{a} 300 \mathrm{~W} / \mathrm{m}^{2}$, podendo estar relacionados com a região (zona equatorial) e o contraste entre oceanos e continentes. Nas análises a seguir, o produto do algoritmo GL1.2 (GOES) será considerado referência para comparação com os demais produtos devido a resultados encontrados em diversos trabalhos onde o desempenho do algoritmo GL1.2 apresenta bons resultados, por exemplo, apresentando erros inferiores a $10 \%$ na media mensal em regiões com pouca carga de aerossóis, Ceballos e Bottino (2004).

A reanálise regional Eta/CPTEC (Figura 3a) superestima a média para toda a América do Sul, atribuindo à maior parte do continente valores acima de $300 \mathrm{~W} / \mathrm{m}^{2}$. Os demais produtos possuem uma espacialização com menores valores das médias, sendo que as reanálises do NCEP/NCAR (Figura 3c) e ERA-40 (Figura 3d) possuem uma razoável concordância nos valores encontrados, principalmente na região central do Brasil, onde ambos registram valores em torno de $250 \mathrm{~W} / \mathrm{m}^{2}$, e sobre os oceanos adjacentes ao continente. As maiores discrepâncias ocorrem na superestimativa do NCEP/NCAR sobre o nordeste brasileiro e sobre o Deserto do Atacama, e na subestimativa de ambas as reanálises na Amazônia.

\subsection{Análise da Variação Meridional}

Na Figura 4 encontra-se representada a variação meridional da radiação solar incidente para os períodos de abril a setembro (Figura 4a) e outubro a março (Figura 4b). Estes períodos

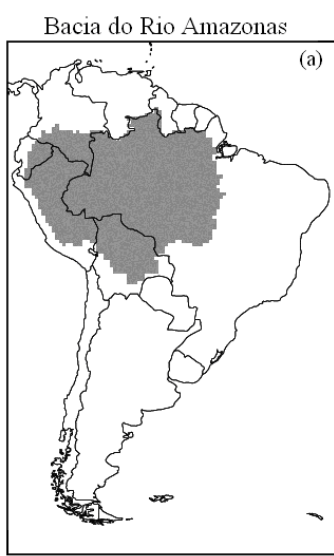

Bacia do Rio São Francisco

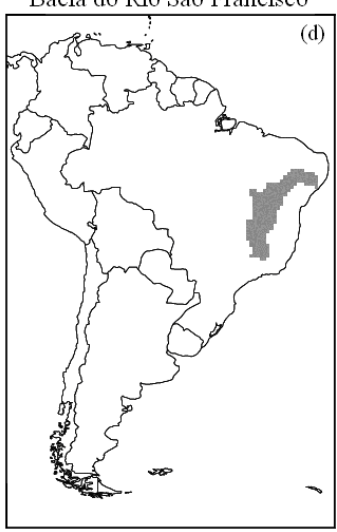

Bacia do Rio Orinoco

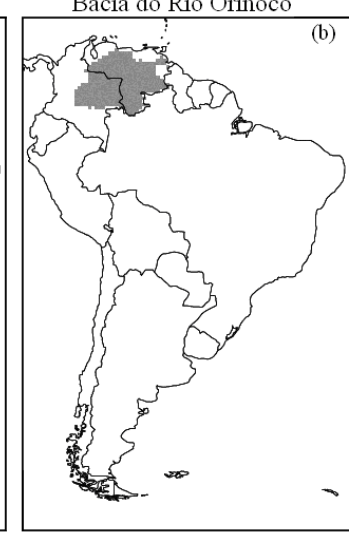

Bacia do Rio Paraná/Prata

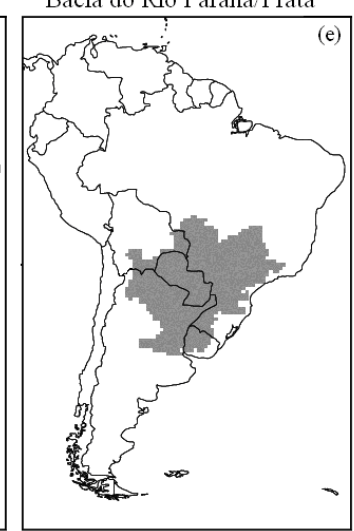

Bacia do Rio Tocantins/Araguaia

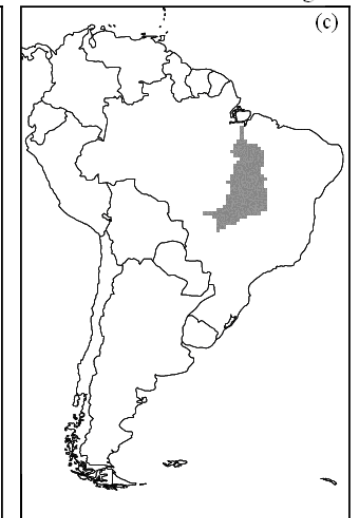

Bacia dos Rios da Patagônia

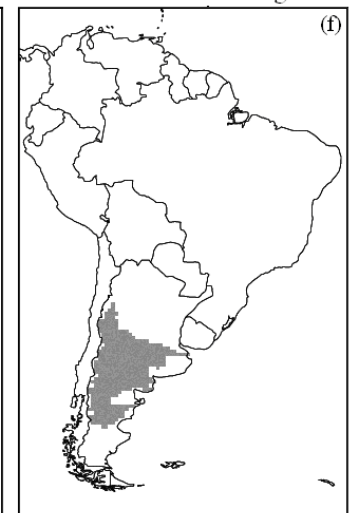

Figura 2 - Máscaras das principais bacias hidrográficas da América do Sul, (a) Bacia do Rio Amazonas, (b) Bacia do Rio Orinoco, (c) Bacia do Rio Tocantins/Araguaia, (d) Bacia do Rio São Francisco, (e) Bacia do Rio Paraná/Prata e (f) Bacia dos Rios da Patagônia. 
foram adotados com a intenção de analisar separadamente o comportamento desta variável para o período centrado no verão (outubro a março) e centrado no inverno (abril a setembro).

$\mathrm{Na}$ Figura $4 \mathrm{a}$ verifica-se que as reanálises do NCEP/ NCAR e ERA-40 apresentam médias com valores bem próximos para todas as bandas e encontram-se dentro do intervalo de confiança (IC) do algoritmo GL1.2 (GOES). Por outro lado, a reanálise Eta/CPTEC superestima as estimativas via satélite para todas as latitudes em relação ao produto do algoritmo GL1.2 (GOES), porém o padrão da variação meridional é também observado para o produto de reanálise Eta/CPTEC.

A Figura $4 \mathrm{~b}$ mostra que existe uma menor variabilidade meridional para os meses centrados no verão, que pode ser observado em todos os produtos. As reanálises do NCEP/NCAR e ERA-40, também apresentam uma boa concordância entre suas médias para todas as latitudes, porém a partir da latitude de $30^{\circ} \mathrm{S}$ seus valores ficam acima do intervalo de confiança do algoritmo
GL1.2 (GOES), superestimando o produto de referência, mas vale a pena ressaltar que para estas latitudes os erros verificados no algoritmo GL1.2 são maiores, conforme os resultados apresentados por Ceballos e Bottino (2004). A reanálise Eta/CPTEC apresenta, durante todo o período, valores médios bem mais altos para todas as bandas, mantendo esses valores acima de $300 \mathrm{~W} / \mathrm{m}^{2}$.

O produto de reanálise regional Eta/CPTEC superestima consideravelmente a radiação em todo o continente tanto no inverno como no verão, embora o perfil da sua curva acompanhe a curva do produto de referência.

\subsection{Análise por Tipo de Vegetação}

Na Figura 5 estão representadas as médias mensais da radiação solar incidente para os principais tipos de vegetação da América do Sul e o intervalo de confiança para o produto do algoritmo GL1.2.
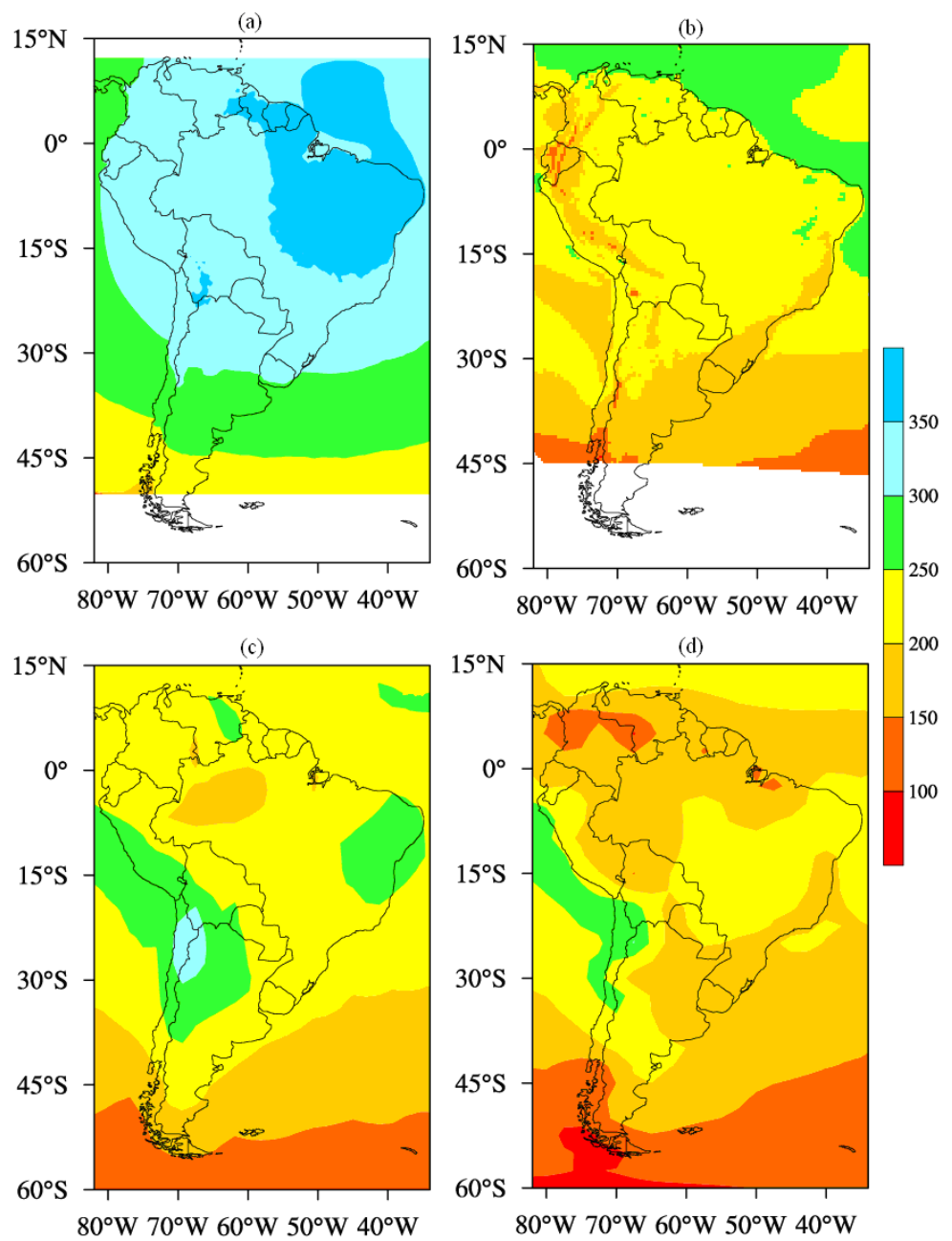

Figura 3 - Climatologia da radiação solar incidente $\left(\mathrm{W} / \mathrm{m}^{2}\right)$ para a América do Sul, (a) reanálise regional Eta/CPTEC, (b) Algoritmo GL1.2 (GOES), (c) reanálise NCEP/NCAR, e (d) reanálise do ERA-40. 
Nas Figuras $5 \mathrm{a}$ e $5 \mathrm{~b}$, observa-se que as reanálises do NCEP/NCAR e ERA-40 possuem médias bem próximas entre si para todos os meses do ano, e estas médias também se encontram dentro do IC do GL1.2. Já o produto de reanálise Eta/CPTEC tem os valores médios mensais superestimados para todo o ano, acima do IC.

No bioma caatinga (Figura 5c), nota-se que as reanálises do NCEP/NCAR e ERA-40 apresentam uma maior diferença entre si, principalmente nos meses de novembro a abril (em torno de $35 \mathrm{~W} / \mathrm{m}^{2}$ ). Esses produtos subestimam o GL1.2 (GOES) para os meses de abril a setembro. A reanálise do ERA-40 não se encontra dentro do IC do GL1.2, para os meses de março a junho e a reanálise do NCEP/NCAR está fora do IC do GL1.2 nos meses de janeiro e dezembro. A reanálise Eta/ CPTEC superestima a média e sazonalidade, eventualmente apresentando valores dentro do IC do GL1.2 (GOES).

Para o cerrado (Figura 5d), os valores das reanálises NCEP/NCAR e ERA-40 possuem médias mensais com uma forte concordância entre elas e encontram-se dentro do IC do GL1.2 para todos os meses do ano. Já a reanálise Eta/CPTEC tem valores mais altos em relação aos demais produtos, porém para os meses de junho e julho as suas médias são bem próximas do limite superior do IC do GL1.2.
$\mathrm{Na}$ Figura 5e, que representa as médias da radiação solar incidente no bioma pampa, os valores das reanálises NCEP/ NCAR e ERA-40 apresentam valores médios praticamente idênticos durante todo o ano. Todavia, observa-se uma pequena superestimativa destas reanálises para os meses de outubro a março, porém dentro da área correspondente ao IC do GL1.2 (GOES). A reanálise Eta/CPTEC apresenta valores mais altos em relação ao produto do algoritmo GL1.2 (GOES), sendo que nos meses de maio a agosto suas médias encontram-se dentro do IC.

A reanálise regional Eta/CPTEC mostra-se sempre com valores bem mais altos em relação ao algoritmo GL1.2 (GOES), deixando evidente que a metodologia utilizada na reanálise do $\mathrm{Eta} / \mathrm{CPTEC}$ possui uma forte tendência de superestimativa dos valores da radiação solar incidente.

Um aspecto importante a se levar em conta é que a radiação incidente em superfície está fortemente relacionada com a nebulosidade. Por exemplo, na região tropical, onde não há muita variação na radiação incidente em função da estação do ano, é muito importante que os modelos levem em conta a nebulosidade, conseqüentemente os modelo que são utilizados para gerar bancos de dados do tipo de reanálise ou GL1.2 apresentam uma boa parametrização de nuvens. Apesar
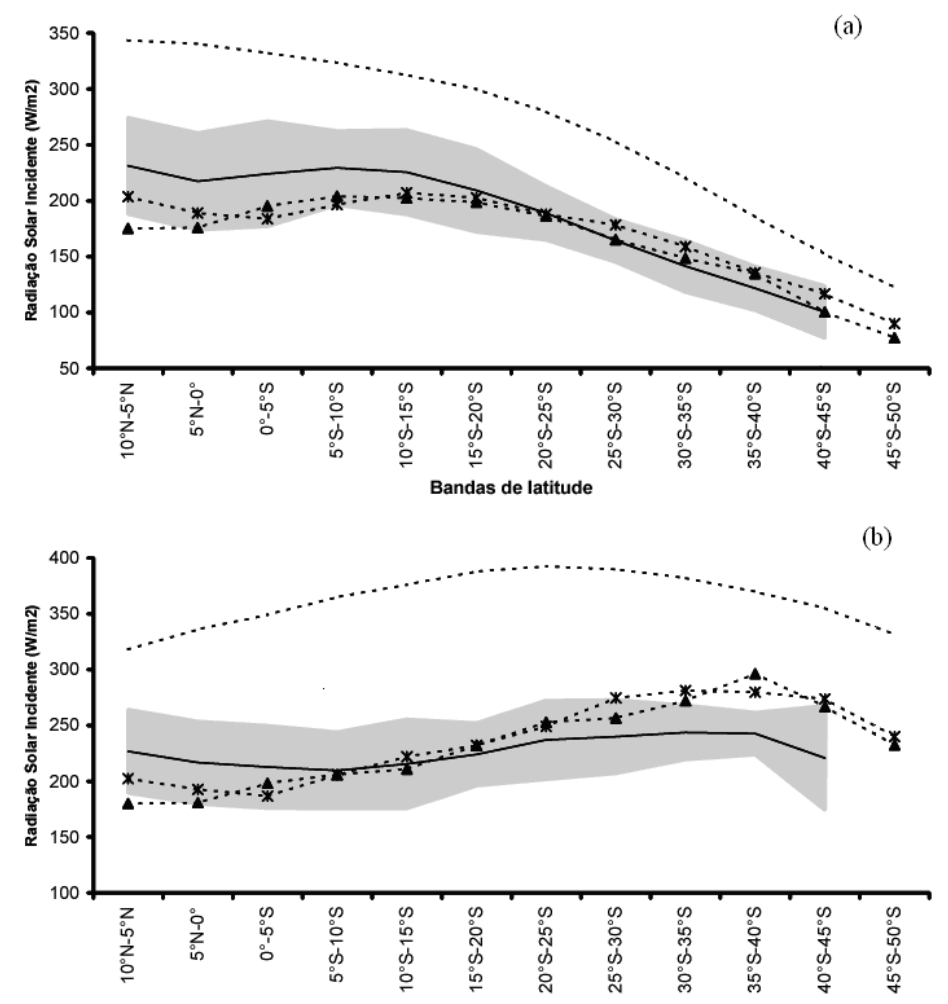

Figura 4 - Variação meridional da radiação solar incidente para a América do Sul, (a) médias para o período de abril a setembro, (b) médias para o período de outubro a março e a área hachurada corresponde ao intervalo de confiança (IC) para a média do produto do algoritmo GL1.2 (GOES) 
da baixa resolução espacial das reanálises do NCEP/NCAR e ERA-40, suas estimativas de nebulosidade parecem bastante consistentes, pelo menos no que diz respeito ao fluxo de radiação solar à superfície.

Um exemplo da importância das nuvens na radiação incidente pode ser observado em diversos experimentos de campo. Correia (2000) mediu a radiação solar incidente na Amazônia no período de 27 de julho a 19 de agosto de 1999 , e encontrou que a radiação solar incidente máxima num determinado período possa variar entre $725 \mathrm{~W} \mathrm{~m}^{-2}$, num dia com nebulosidade de 0,35 e com precipitação, a $1076 \mathrm{~W} \mathrm{~m}^{-2}$, num dia típico da estação seca na região, isto é, com pouca nebulosidade e forte insolação $(0,63)$.

(a) Floresta Tropical Perene

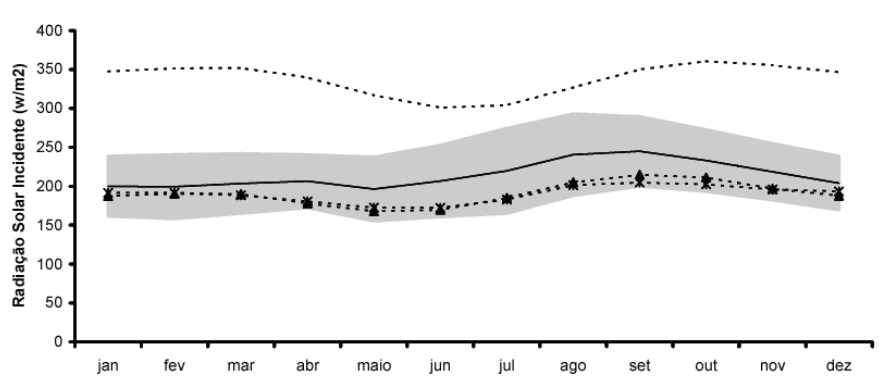

(c) Caatinga Nordeste Brasileiro

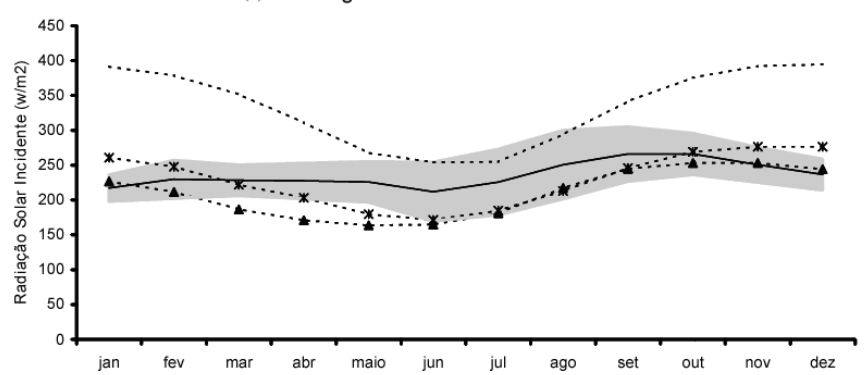

(e) Pampas/Campos

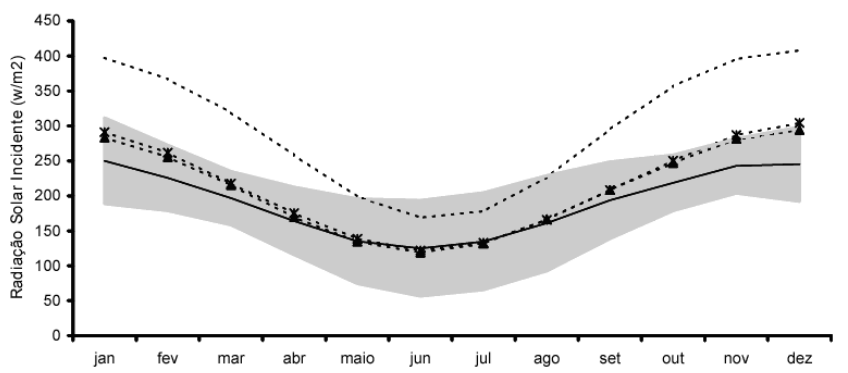

\subsection{Análise para as Principais Bacias Hidrográficas}

Uma visão geral das médias mensais para as principais bacias hidrográficas da América do Sul pode ser vista nos gráficos da Figura 6,onde na bacia do rio Amazonas, as reanálises do NCEP/NCAR e ERA-40 subestimaram ligeiramente as estimativas do GL1.2 (GOES), ainda dentro do seu IC, e com a sazonalidade correta. $\mathrm{Na}$ bacia do rio Orinoco (Figura 6b), as médias do NCEP/NCAR e ERA-40 possuem uma diferença maior entre si, e as médias mensais da reanálise do ERA-40 ficaram abaixo do IC do GL1.2 (GOES) na maioria dos meses.

Para a bacia do rio Tocantins/Araguaia (Figura 6c), as reanálises do NCEP/NCAR e ERA-40 subestimaram as
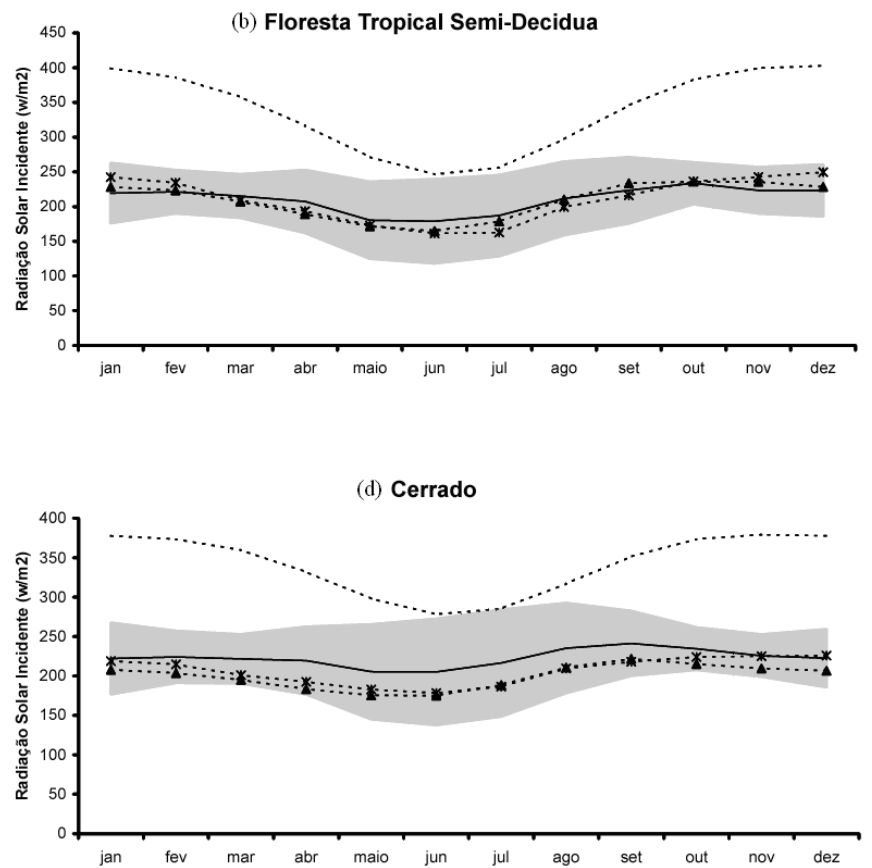

Figura 5 - Médias mensais para a radiação solar incidente para os principais tipos de vegetação da América do Sul, (a) Floresta Tropical Perene, (b) Floresta Tropical Semi-Decídua, (c) Caatinga, (d) Cerrado, (e) Pampas/Campos e a área hachurada corresponde ao intervalo de confiança (IC) para o produto GL1.2 (GOES). 
(a) Bacia do Rio Amazonas

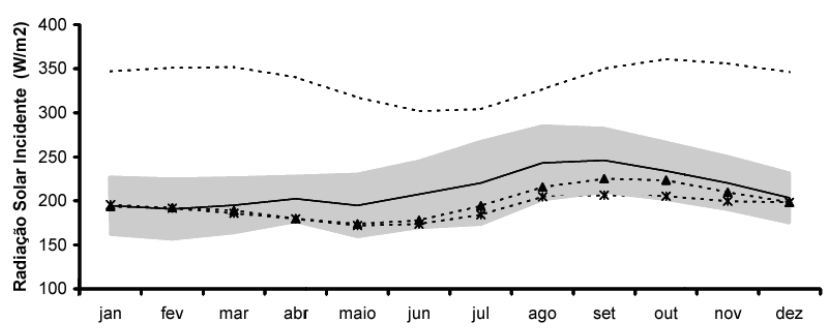

(c) Bacia do Rio Tocantins/Araguaia
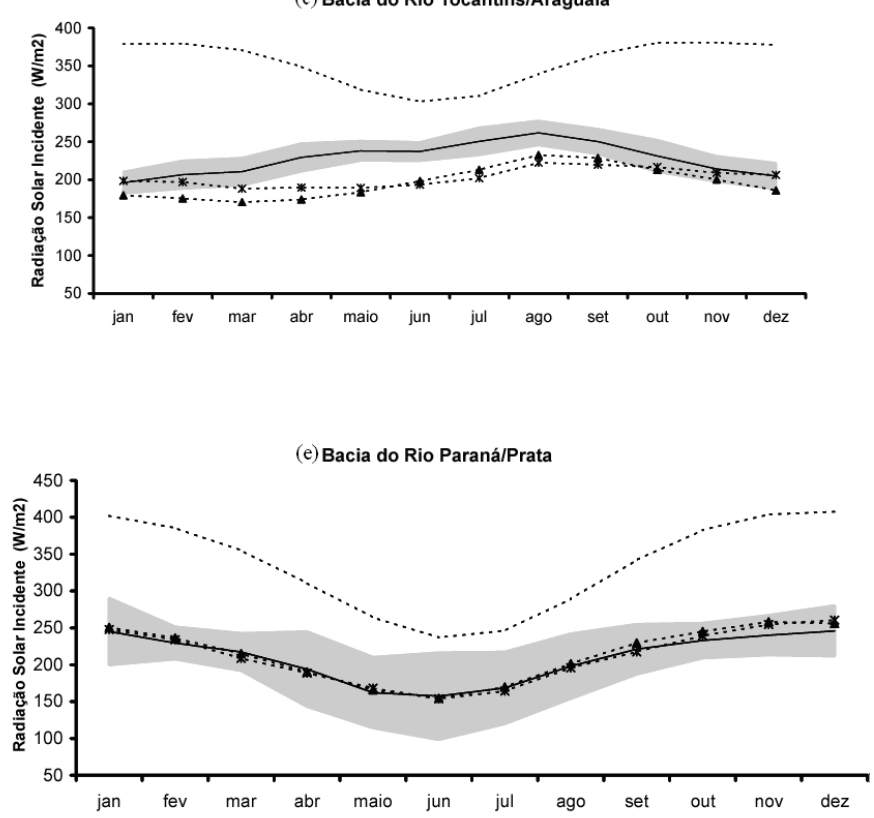

\begin{tabular}{|ll}
\hline$\cdots \cdots$ CPTEC & $\cdots * \cdots$ NCEP/NCAR \\
$\cdots * \cdots$ ERA -40 & - GL1.2 (GOES)
\end{tabular}

(b) Bacia do Rio Orinoco

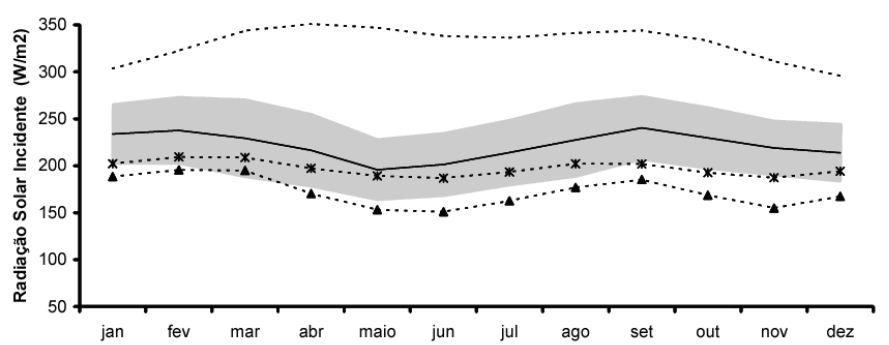

(d) Bacia do Rio São Francisco
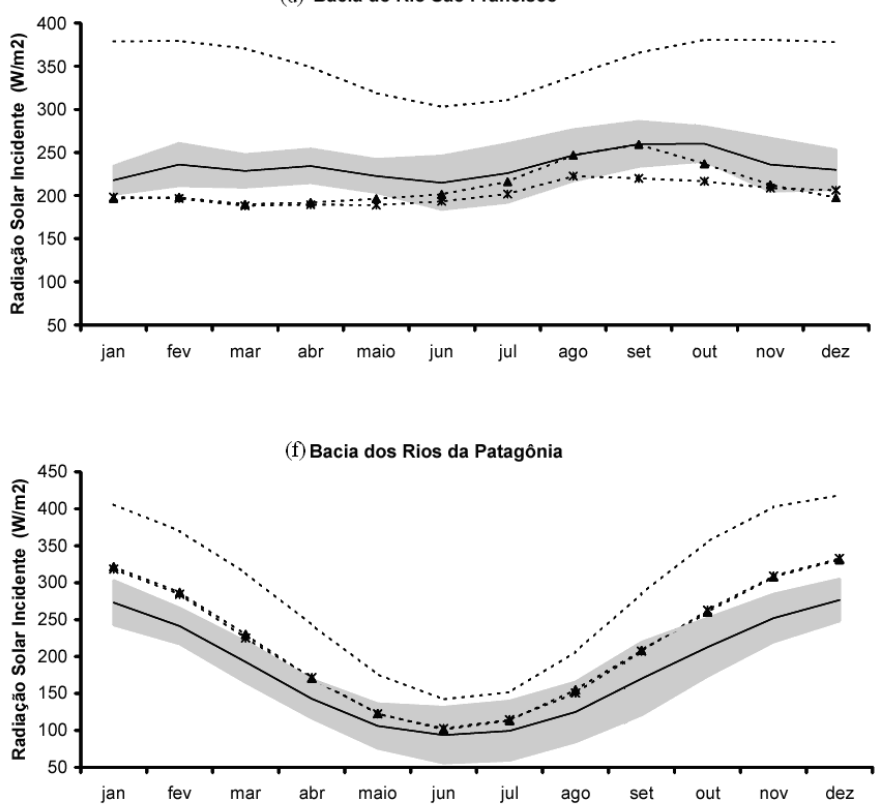

GL1.2 (GOES)

Figura 6 - Médias mensais para a radiação solar incidente para as principais bacias hidrográficas da América do Sul, (a) bacia do Rio Amazonas, (b) bacia do Rio Orinoco, (c) bacia do Rio Tocantins/Araguaia, (d) bacia do Rio São Francisco, (e) bacia do Rio Paraná/Prata, (f) bacia dos Rios da Patagônia e a área hachurada corresponde ao intervalo de confiança (IC) para o produto GL1.2 (GOES).

estimativas do algoritmo GL1.2 (GOES). Nesta bacia o IC teve uma faixa bem estreita, o que ocasionou que em vários meses nenhum dos produtos analisados ficassem dentro do IC do algoritmo GL1.2 (GOES).

A Figura 6d mostra que, na Bacia do rio São Francisco, nos meses de agosto a outubro as reanálises do NCEP/NCAR e ERA-40 possuem as médias um pouco mais afastadas, e no resto dos meses há uma boa concordância entre as médias destes produtos. O IC não abrange estas reanálises nos meses de fevereiro a abril, e de setembro a outubro para a reanálise do ERA-40.

A bacia do rio Paraná/Prata (Figura 6e) e a bacia dos rios da Patagônia (Figura 6f) apresentam valores das duas reanálises (NCEP/NCAR e ERA-40) praticamente iguais entre si. Nestas duas bacias se nota uma sazonalidade maior na radiação, enquanto nas demais bacias os valores médios variam muito pouco no decorrer do ano.

Para as análises realizadas nesta seção, nota-se que a radiação solar incidente obtida pela reanálise do Eta/CPTEC, manteve seu padrão de superestimativa, e em todas as bacias analisadas este produto apresentou médias fora do intervalo de confiança do algoritmo GL1.2 (GOES), e em praticamente todos os casos a sazonalidade esteve exagerada ou mesmo fora de fase.

\section{CONCLUSÕES}

Dentre os produtos de radiação solar incidente à superfície verificou-se que, para a comparação realizada para os tipos de vegetação e bacias hidrográficas, há uma grande 
concordância entre os valores das reanálises do NCEP/NCAR e ERA-40, e na maioria dos meses estes produtos se encontram dentro do IC da média do algoritmo GL1.2 (GOES), o que nos permite concluir, de maneira geral, que estas reanálises possuem uma boa representação da espacialização da radiação solar incidente para diversas regiões do continente.

Para os quatro produtos de radiação solar incidente analisados, nota-se que três [NCEP/NCAR, ERA-40 e algoritmo GL1.2 (GOES)] apresentam valores próximos entre si, e que a reanálise do Eta/CPTEC sempre apresenta valores bem mais elevados. Os dados do satélite GOES, geralmente apresentam resultados bem próximos das demais reanálises, porém em alguns casos isolados as diferenças chegam a $60 \mathrm{~W} / \mathrm{m}^{2}$ (bacia do rio Orinoco).

No caso do produto de reanálise Eta/CPTEC ficou clara a sua deficiência em estimar a radiação solar incidente à superfície. Tarasova et al. (2006) implantaram uma nova parametrização para o regime de radiação solar no modelo regional Eta, a fim de melhorar a representação do fluxo em superfície e avaliar as alterações que ocorrem nas distintas variáveis meteorológicas do modelo, onde verificaram que a nova parametrização da radiação demonstrou melhores resultados tanto para condições de céu claro como nublado. Espera-se que esta reanálise Eta/ CPTEC tenha sido gerada utilizando o novo código de regime de radiação solar. Esta deficiência apresentada por este produto pode estar relacionada com a parametrização de nuvens no modelo Eta usado pelo CPTEC, ou até mesmo com o método utilizado pelo centro para criar este banco de dados de reanálise.

\section{REFERÊNCIAS BIBLIOGRÁFICAS}

ARAKAWA, A.; V. R. LAMB. Computational design of the basic dynamical processes of the UCLA general circulation model. Methods in Computational Physics, v. 17, p. 173265, 1977.

BLACK T. L. The new NMC mesoscale Eta model: Description and forecast examples. Journal Weather and Forecasting, v. 9, p. 265-278, 1994.

BASTOS, E. J. B. et al. Estimativa da radiação solar Global para a América do Sul via Satélite. In: Anais, Congresso Brasileiro de Meteorologia, Campos do Jordão, p.596-600, 1996.

CALVET, J. C.; Y. VISWANADHAM. Amazon Forest Radiation Budget from Satellite Data. Journal of Applied Meteorology, v. 32, p. 844-847, 1993.

CEBAlloS, J. C.; G. B. MOURA. A.Solar Radiation Assessment Using Meteosat 4-VIS Imagery. Solar Energy, v. 60, p. 209-219, 1997.

CEBAllos, J. C. e M. J. BOTTINO. Estimativa de radiação solar por satélite: Desempenho do modelo operacional GL1.2. In: Anais, XIII Congresso Brasileiro de Meteorologia, Fortaleza, 2004.

CEBALLOS, J.C.; M. J. BOTTINO; J. M. de SOUZA. A simplified physical model for assessing solar radiation over Brazil using GOES 8 visible imagery. Journal of Geophysical Research, v. 109, D02211, doi:10.1029/2003JD003531, 2004. CORREIA, F. W. S. Estudo do Balanço de Radiação em Área de Policultivo na Amazônia. 137p. Dissertação (Mestrado em Meteorologia) - Instituto Nacional de Pesquisas Espaciais, São José dos Campos, 2000.

GALVÃO, J. A. C. Estudo do balanço de radiação em áreas de floresta e pastagem na Amazônia. 125p. Dissertação (Mestrado em Meteorologia) - Instituto Nacional de Pesquisas Espaciais, São José dos Campos, 1999.

JANJI'C, Z. I. Forward-backward scheme modified to prevent two-grid-interval noise and its application in sigma coordinate models. Contributions to Atmospheric Physics, v. 52, p. 69-84, 1979.

KALNAY, E. et al. The NCEP/NCAR 40-Year Reanalysis project. Bulletin of the American Meteorological Society, v. 77, p. 437-471, 1996.

KÅLLBERG, P. et al. ERA-40 Project Report Series No. 17. The ERA-40 archive, 2004.

MESINGER, F. An economical explicit scheme which inherently prevents the false two-grid-interval wave in the forecast fields, Symp. "Difference and Spectral Methods for Atmosphere and Ocean Dynamics Problems", Academy of Sciences, Novosibirsk 1973, Part II, p. 18-34, 1974.

MESINGER, F. et al. The step-mountain coordinate: Model description description and performance for cases of Alpine lee cyclogenesis and for a case of Appalachian redevelopment. Journal Monthly Weather Review, v.116, p. 1493-1518, 1988.

POHLMANN, H.; R. J. GREATBATCH. Discontinuities in the late 1960's in different atmospheric data products. Geophysical Research Letters, v. 33, L22803, doi:10.1029/2006GL027644, 2006.

ROSSOW, W. B.; Y. C.ZHANG. Calculation of Surface and Top of Atmosphere Radiative Fluxes from Physical Quantities Based on ISCCP Data Sets 2. Validation and First Results. Journal of Geophysical Research, v. 100, p. 1167-1197, 1995.

TARASOVA, T. A. et al. Impact of new solar radiation parameterization in the Eta model on the simulation of summer climate over South America. Journal of Applied Meteorology and Climatology, v. 45, n. 2, p. 318-333, 2006.

ZHANG, Y. C.; W. B. ROSSOW; A. A. LACIS. Calculation of Surface and Top of Atmosphere Radiative Fluxes from Physical Quantities Based on ISCCP Data Sets 1. Method and Sensivity to Input Data Uncertainties. Journal of Geophysical Research, v. 100, p. 1149-1165, 1995. 